\title{
SOME SHORT ELEMENTS ON HEDGING CREDIT DERIVATIVES *
}

\author{
Philippe Durand ${ }^{1}$ AND JEAN-FrÉDÉRIC JOUANIN ${ }^{1}$
}

\begin{abstract}
In practice, it is well known that hedging a derivative instrument can never be perfect. In the case of credit derivatives (e.g. synthetic CDO tranche products), a trader will have to face some specific difficulties. The first one is the inconsistence between most of the existing pricing models, where the risk is the occurrence of defaults, and the real hedging strategy, where the trader will protect his portfolio against small CDS spread movements. The second one, which is the main subject of this paper, is the consequence of a wrong estimation of some parameters specific to credit derivatives such as recovery rates or correlation coefficients. We find here an approximation of the distribution under the historical probability of the final Profit \& Loss of a portfolio hedged with wrong estimations of these parameters. In particular, it will depend on a ratio between the square root of the historical default probability and the risk-neutral default probability. This result is quite general and not specific to a given pricing model.
\end{abstract}

Mathematics Subject Classification. 91B28.

Invited paper accepted September 2005.

\section{INTRODUCTION}

The widespread use of models for pricing exotic options introduces some new parameters that cannot always be calibrated on available market data, and therefore may induce some model risk. When selling a derivative instrument, the price of which depends on such a coefficient, a technique commonly used by traders is to give that unknown parameter a value that ensures a conservative price and keep this value along time to hedge themselves. This is for example the case for an equity derivative trader who does not precisely know the level of the volatility of one asset, but assumes that it will remain in some prescribed interval. Whenever he sells a call, he will overestimate his price using as volatility the upper boundary of the interval and keep this level to hedge himself. This heuristic technique may also be used by an exotic interest rate derivative trader, when having a sensitivity with respect to a long-term volatility or a correlation coefficient between two long-term swap rates.

In the case of equity derivatives, this basic intuition was justified in El Karoui, Jeanblanc-Picqué and Shreve [4]. The authors show that the Black-Scholes pricing formula is robust with respect to a misspecification of the volatility. To be more specific, when a trader sells an option which he prices and replicates within a local volatility model 'à la Dupire' [3] (which includes the Black-Scholes case), if the option payoff is convex

Keywords and phrases. Credit derivatives, hedging, robustness.

* The authors would like to thank Nicole El Karoui, Mathieu Bernardo, Gaël Riboulet and Roberto Silviotti for helpful discussions. This work was completed when the authors worked at Crédit Lyonnais. All remaining errors are ours.

${ }^{1}$ Natixis, 115 rue Montmartre, F-75002 Paris, France; philippe.durand@polytechnique.org; jean-frederic.jouanin@ponts.org 
and if the local volatility process dominates the (unknown) true volatility, then the value of the misspecified hedging strategy will dominate the value of the option at expiration. This holds even if the actual volatility is stochastic.

As regarding credit derivatives, the intuition of many traders is that the extension of that result should let appear the historical default probabilities. Indeed, if one hedges for example a synthetic CDO tranche - in continuous time and more generally assuming that the hypothesis of the model used fits perfectly to the reality - using some wrong values of recovery rates or correlation coefficients, and if no default occurs during the life of the product, the portfolio should be perfectly self-financed. Here we try to confirm - or infirm - this intuition. Of course, in the real world, a wrong estimation of a correlation parameter will also modify the gamma of the portfolio and therefore will affect the error due to a discrete-time rebalancing. This point will not be studied here, being not specific to credit derivatives.

The paper is organized as follows. We will first recall some basics on the pricing of credit derivatives, and in particular discuss the consequence of using a model such as a copula model where the source of risk is the occurrence of defaults and hedging against small margin movements. We will then study what happens when using a wrong estimation of a recovery rate or of a correlation coefficient. In both cases we obtain some estimations of the distribution of the P\&L of a portfolio hedged using wrong estimations of these parameters.

\section{General FRAMEWORK OF THE DisCUSSION}

\subsection{Some general facts on credit derivatives}

One cannot address the pricing and hedging of credit derivatives, and especially CDO tranches, without at least evoking two most important points:

- the "base" correlation skew: now that some liquidity has been achieved on the market of CDO tranches for a few standard portfolios (iTraxx Europe, CDX North America, etc.), credit derivatives traders have become used with quoting the tranches with implied Gaussian one-factor copula correlation coefficients, in the same way that equity calls or interest-rate caplets are quoted with Black volatilities. This has resulted in the observation of a market correlation skew. This topic is the main subject of recent publications about the pricing of CDO tranches, and many authors try to find a model of the dependence of defaults consistent with this observation (see for example the random factor-loading model of Andersen and Sidenius [1]);

- the market incompleteness: the full mathematical study of the hedging of credit derivatives (at least with one issuer) was carried out by Jeanblanc and Rutkowski [5] and Bielecki and Jeanblanc [2]. They discuss the possibility for the trader of replicating a credit derivative depending on the complexity of the pay-off (e.g. with zero or non-zero recovery rate) and on the set of financial instruments available.

We do not intend to fully discuss these two points, but we now give a short illustration of the last one.

For each underlying obligor, a successful trader should hedge his position against at least two risks: the occurrence of a default, and if not, a variation of the credit spreads. What kind of CDS-based strategy should he follow?

- If he hedges himself against defaults only, his portfolio is not protected against CDS spread movements, therefore he cannot keep his P\&L from one day to another.

- If he tries to protect himself against both risks by using, for example, two CDS with different maturities, he is likely to find himself in a more toxic situation than with a standard, albeit imperfect, hedge. Let us consider he bought a protection on a mezzanine tranche of a five-year maturity CDO tranche. To hedge himself against both risks using a $3 \mathrm{Y}$ and a $5 \mathrm{Y}$ CDS, on practical examples, he will buy a $3 \mathrm{Y}$ protection and sell a $5 \mathrm{Y}$ protection, the latter for a higher notional than for a simple jump-to-default or margin movements hedge. As time goes by, suppose the quality of one credit weakens: the $3 \mathrm{Y}$ and 
$5 Y$ CDS spreads of this name explode and the bid-ask spread widens. When the short-term CDS is close to expiry, either the trader manages to roll this protection and pays extremely expensive liquidity costs, or he does nothing and gets in an extremely risky position.

- Finally, the more realistic strategy seems to be to hedge against spread movements only when no default is expected in the near future, and progressively change to a hedging strategy against default if one credit weakens.

However the consequences of this choice are not neutral. Indeed, our trader applies a hedging strategy which is not consistent at all with the hypothesis of the pricing model used. And as, in the latter, the unique source of risk is the occurrence of a credit event, the theory tells us that only a portfolio hedged against defaults should be replicating. A portfolio hedged against spread movements will not be protected against a default and will have have a - positive or negative - time decay (theta). One can make an analogy with an option on an asset priced with the Black-Scholes formula, and hedged with the delta and the vega. In the Black-Scholes model, if we suppose the volatility deterministic, the theory shows us that a delta-hedged portfolio will be self-financing. Such a hedging strategy needs to be complemented by a vega hedge because of the discrepancy between the hypothesis and the reality, and is justified by the Taylor formula only. In the case of credit derivatives, the first risk is the occurrence of a default (a movement of the price of the underlying equity), and the second is a variation of its default probability (its credit spread) (a movement of the volatility of the asset). Hedging a complex credit derivative against margin movements and not against credit events would be like hedging an option on an asset using Black-Scholes vega and not delta.

\subsection{General framework and notations}

In the remainder of the paper, the following notations and assumptions will be in force. We consider a universe of $d$ credit issuers, the default times of which are noted $\tau_{i}$ and defined as

$$
\tau_{i}:=\inf \left\{t \geq 0: \int_{0}^{t} \lambda_{i}(s) \mathrm{d} s>\theta_{i}\right\},
$$

where each function $\lambda_{i}$ is "càdlàg" and describes the intensity of the corresponding issuer $i^{1}$. The random variables $\left(\theta_{i}, i=1, \ldots, d\right)$ follow the standard exponential distribution but are - in general - not independent. The joint distribution of these variables can be written as

$$
\mathbb{Q}\left(\theta_{1}>x_{1}, \ldots, \theta_{d}>x_{d}\right)=C\left(\mathrm{e}^{-x_{1}}, \ldots, \mathrm{e}^{-x_{d}}\right), \quad \forall x_{1}, \ldots, x_{d} \geq 0
$$

The function $C:[0,1]^{d} \rightarrow[0,1]$ is a copula, hence the name of "intensity-based copula framework" given to this kind of model, which has become classical for modelling multi-name credit derivatives since the paper of Schönbucher and Schubert [7]. In their setting, the intensities are stochastic processes - independent from the random vector $\left(\theta_{i}, i=1, \ldots, d\right)$ - and the market filtration is built by enlargement. We will not address this subject here [6]. The most common copula is the one-factor Gaussian copula which is used as a "Black" standard in the market to quote the CDO tranches, despite it gives rise to some difficulties (correlation skew, time-inhomogeneity). In case of default of issuer $i$, the owner of a bond issued by $i$ receives only a fraction $R_{i}$ of the initial notional of the bond, which is the recovery rate.

We make the usual hypothesis that all the recovery rates are deterministic, since we do not have any market information to calibrate distributions. We also assume zero interest rates to simplify the notations, since they lead to very tiny sensitivities for most credit derivatives instruments. For a probability measure $\mathbb{P}$ we will

\footnotetext{
${ }^{1}$ The intensity is calibrated on the term-structure of default probabilities through the equation
}

$$
\mathbb{Q}_{t}\left(\tau_{i} \leq T\right)=1-\exp \left(-\int_{t}^{T} \lambda_{i}(s) \mathrm{d} s\right) \text { on }\left\{\tau_{i}>t\right\} .
$$


denote by $\mathbb{P}_{t}(A)$ and $\mathbb{E}_{t}^{\mathbb{P}}(X)$ the conditional probability of the event $A$ and the conditional expectation of $X$ with respect to the market information (included the default processes) up to time $t$. Likewise, $\sigma^{\mathbb{P}}(X)$ will stand for the standard deviation of $X$ under $\mathbb{P} . \mathbb{Q}$ (resp. $\mathbb{P}$ ) will denote the risk-neutral (resp. historical) probability measure. Finally, we state an important result:

Proposition 1.1. Under the preceding model assumptions, the credit market is complete under $\mathbb{Q}$.

Proof. This is straightforward if we look at the default process as a marked point process whose increasing family of random times is the successive default times and whose marks are the names of the defaulting issuers. The proposition stems from the martingale representation theorem for (marked) point processes.

When replicating an instrument on the equity market, the ratios invested in the risky assets are proportional to the derivatives of the price with respect to the assets, but here they depend on the jumps of the price - which the market call jumps-to-default - in case of a default of any issuer.

\section{ERroneous RECOVERY RAte}

\subsection{Case of one credit}

Consider a single-name credit derivative (called $C D$ ) of maturity $T$ with an up-front payment only. We drop subscripts in the notations of the preceding subsection and denote by $f(R)$ the payoff of the exotic product at default time $\tau$. We recall that we consider zero interest rates.

We do have the price at time $t \leq T$ (on the event $\{\tau>t\}$ ) of the exotic product:

$$
C D_{t}=\mathbb{E}_{t}^{\mathbb{Q}}\left[f(R) 1_{\{\tau \leq T\}}\right]=f(R) \mathbb{Q}_{t}[\tau \leq T]
$$

and of a simple (up-front payment only) $C D S$ with the same maturity:

$$
C D S_{t}=\mathbb{E}_{t}^{\mathbb{Q}}\left[(1-R) 1_{\{\tau \leq T\}}\right]=(1-R) \mathbb{Q}_{t}[\tau \leq T]
$$

Let us (statically) hedge against default the $C D$ with a quantity $\delta$ of $C D S$. If the issuer defaults at time $t<T$ with the recovery rate $R$, we will observe some jumps on the prices of the $C D$ and of the $C D S$ :

$$
\begin{aligned}
\text { jump }_{t}^{C D} & =f(R)-C D_{t-} \\
& =f(R)\left(1-\mathbb{Q}_{t-}[\tau \leq T]\right) \\
\text { jump }_{t}^{C D S} & =(1-R)-C D S_{t-} \\
& =(1-R)\left(1-\mathbb{Q}_{t-}[\tau \leq T]\right) .
\end{aligned}
$$

The replicating CDS ratio $\delta$ is such that:

$$
j u m p_{t}^{C D}+\delta j u m p_{t}^{C D S}=0, \quad \forall t \leq T
$$

which gives:

$$
\delta(R)=-\frac{f(R)}{1-R}
$$

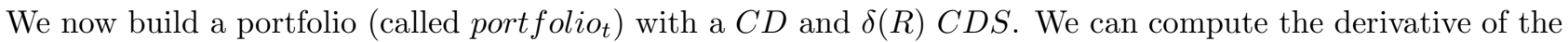
price of portfolio $t$ with respect to the recovery rate $R$, with the market price $C D S_{t}$ unchanged $^{2}$ and also the

\footnotetext{
${ }^{2}$ This means that when we shift the recovery rate $R$, we perform a re-calibration of the default probabilities, before re-computing the price of the portfolio. This is consistent with the market practice.
} 
composition of portfolio $($ that is $\delta$ ) unchanged. Here we assume that $f$ is smooth:

$$
\left.\frac{\partial \text { portfolio }}{\partial R}\right|_{\substack{\text { portfolio composition unchanged } \\ C D S_{t} \text { unchanged }}}=\left(f^{\prime}(R)+\frac{f(R)}{1-R}\right) \mathbb{Q}_{t}[\tau \leq T] .
$$

Finally we study the P\&L of portfolio $t$ when a default occurs, with a realized recovery rate $R$ and a misspecified recovery assumption $\hat{R}$. The observed jumps of the $C D$ and the $C D S$ still are:

$$
\begin{aligned}
\text { jump }_{t}^{C D} & =f(R)-C D_{t-} \\
\text { jumpt }_{t}^{C D S} & =(1-R)-C D S_{t-}
\end{aligned}
$$

but the jump of the NPV of the global portfolio becomes if the default occurs at time $t \leq T$ :

$$
\begin{aligned}
j u m p_{t}^{\text {total }} & =f(R)-f(\hat{R}) \frac{1-R}{1-\hat{R}} \\
& =\left(f^{\prime}(R)+\frac{f(\hat{R})}{1-\hat{R}}\right)(R-\hat{R})+o(R-\hat{R}) \\
& =\left.\frac{\mathbf{1}_{\tau \leq T}}{\mathbb{Q}_{t_{-}}[\tau \leq T]} \frac{\partial C D_{t-}}{\partial R}\right|_{C D S_{t-}}(R-\hat{R})+o(R-\hat{R}) .
\end{aligned}
$$

The P\&L at date $T$ of the global portfolio is finally:

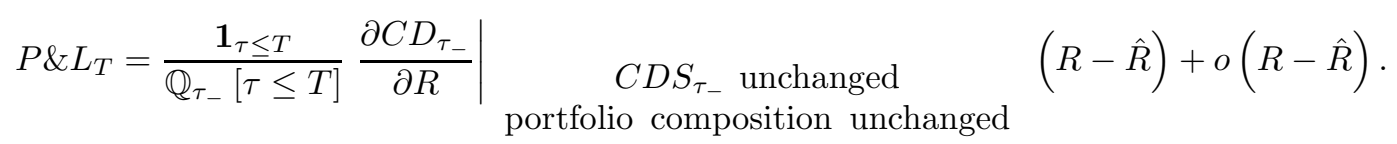

If we know the distribution of the "real" recovery rate under the historical probability, we then have an approximation of the distribution of the P\&L of the portfolio. We can state:

Approximation result 2.1. Let us assume that:

- $\left.\frac{\partial C D_{t}}{\partial R}\right|_{C D S_{t}}$ is nearly constant;

- $C D S$ margins are flat;

- the default event and the error on the estimation of the recovery rate independent.

we have the approximation:

$$
\left.\mathbb{E}^{\mathbb{P}}\left[P \& L_{T}\right] \simeq \frac{\mathbb{P}[\tau \leq T]}{\mathbb{Q}[\tau \leq T]} \frac{\partial C D}{\partial R}\right|_{C D S}\left(\mathbb{E}^{\mathbb{P}}[R]-\hat{R}\right)
$$

If we further suppose that $R$ follows a Gaussian distribution under the historical probability $\mathbb{P}$, with a mean $\hat{R}$, which is the misspecified value used by the trader, and with a standard deviation $\sigma^{\mathbb{P}}(R)$, then the distribution of the PELL at expiry $T$ under $\mathbb{P}$ has the following properties:

$$
\begin{aligned}
\mathbb{E}^{\mathbb{P}}\left[P \& L_{T}\right] & \simeq 0 \\
\sigma^{\mathbb{P}}\left[P \& L_{T}\right] & \simeq \frac{\sqrt{\mathbb{P}[\tau \leq T]}}{\mathbb{Q}[\tau \leq T]}\left|\frac{\partial p o r t \text { folio }}{\partial R}\right| \begin{array}{c}
C D S \text { unchanged } \\
\text { portfolio composition unchanged }
\end{array} \mid \sigma^{\mathbb{P}}(R) .
\end{aligned}
$$




\section{Numerical application:}

- $T=5$ years;

- $\hat{R}=30 \%$;

- $\mathbb{P}[\tau \leq T]=1.25 \%$ (S\&P data, for an issuer between $\mathrm{A}$ and $\mathrm{BBB}$ );

- $C D S$ margin $=150 \mathrm{bp}$.

We find $\mathbb{Q}[\tau \leq T] \simeq 10 \%$ and $\frac{\sqrt{\mathbb{P}[\tau \leq T]}}{\mathbb{Q}[\tau \leq T]} \simeq 1.1$.

\subsection{Case of $d$ credits}

We now consider a basket of $d$ credits. First we suppose we perfectly know the recovery rates of the $d-1$ last ones. Given the default times of credits 2 to $d$, the previous subsection gives us the conditional distribution of the final $P \& L$ due to an error on the recovery rate of the first credit. But here, we may hedge perfectly against the defaults of credits 2 to $d$, therefore the distribution of the final $P \& L$ will not depend on the default time of credits 2 to $d$. The result of the previous section gives us then the distribution of the final $P \& L$.

We now suppose that we have uncertainties on all recovery rates $R_{i}$. For the sake of simplicity, we suppose that the ratio $\frac{\sqrt{\mathbb{P}\left[\tau_{i} \leq T\right]}}{\mathbb{Q}\left[\tau_{i} \leq T\right]}$ and the standard deviations $\sigma^{\mathbb{P}}\left(R_{i}\right)$ are the same for all different credits. We again drop the subscript $i$ accordingly. We state:

Approximation result 2.2. Under the preceding assumptions, the distribution of the P\&SL at expiry $T$ under $\mathbb{P}$ has the following properties:

$$
\mathbb{E}^{\mathbb{P}}\left[P \& L_{T}\right] \simeq 0
$$

and for the standard deviation:

- with a 100\%-correlation between the misspecifications of recovery rates but no link between occurrences of default:

$$
\sigma^{\mathbb{P}}\left[P \& L_{T}\right] \simeq \frac{\sqrt{\mathbb{P}[\tau \leq T]}}{\mathbb{Q}[\tau \leq T]}\left|\sum_{k=1}^{d} \frac{\partial p o r t \text { folio }}{\partial R_{k}}\right| \begin{gathered}
C D S \text { unchanged } \\
\text { portfolio composition unchanged }
\end{gathered} \mid \sigma^{\mathbb{P}}(R)
$$

- in the worst case for the misspecification of recovery rates, and no link between occurrences of default:

$$
\sigma^{\mathbb{P}}\left[P \& L_{T}\right] \simeq \frac{\sqrt{\mathbb{P}[\tau \leq T]}}{\mathbb{Q}[\tau \leq T]}\left(\sum_{k=1}^{d}\left|\frac{\partial \text { portfolio }}{\partial R_{k}}\right| \begin{array}{c}
C D S \text { unchanged } \\
\text { portfolio composition unchanged }
\end{array} \mid\right) \sigma^{\mathbb{P}}(R)
$$

- with a 0\%-correlation between the misspecifications of recovery rates and still no link between occurrence of defaults:

$$
\sigma^{\mathbb{P}}\left[P \& L_{T}\right] \simeq \frac{\sqrt{\mathbb{P}[\tau \leq T]}}{\mathbb{Q}[\tau \leq T]} \sqrt{\sum_{k=1}^{d}\left(\frac{\partial \text { portfolio }}{\partial R_{k}} \mid \begin{array}{c}
C D S \text { unchanged } \\
\text { portfolio composition unchanged }
\end{array}\right)^{2}} \sigma^{\mathbb{P}}(R) .
$$




\section{ERroneous CORRELATiOn COEFFICIENT}

\subsection{Case of 2 issuers}

We study the case of a product on a basket of two issuers, $A$ and $B$. The exotic product (still denoted $C D$, typically a first-to-default product), delivers a flow $F_{1}$ when a first default occurs before $T$ (independent of which issuer defaults), a flow $F_{2}$ when a second default occurs again before $T$ and zero otherwise. We assume that the joint default probability of the two issuers depends on an (unknown) parameter $\rho$. To simplify the calculations, we consider zero recovery rates.

We denote:

- $\tau_{A}$ the default time of issuer $A$;

- $\tau_{B}$ the default time of issuer $B$;

- $f_{A}(\rho):=\mathbb{Q}_{t}\left[\tau_{B}<T \mid \tau_{A}<T, \tau_{A}<\tau_{B}\right]^{3}$;

- $f_{B}(\rho):=\mathbb{Q}_{t}\left[\tau_{A}<T \mid \tau_{B}<T, \tau_{B}<\tau_{A}\right]$;

- $g_{A}(\rho):=\mathbb{Q}_{t}\left[\tau_{A}<T, \tau_{A}<\tau_{B}\right]$;

- $g_{B}(\rho):=\mathbb{Q}_{t}\left[\tau_{B}<T, \tau_{B}<\tau_{A}\right]^{4}$.

We can summarize this in the "transition probability" matrix under $\mathbb{Q}^{5}$ :

\begin{tabular}{|c|cccc|}
\hline & A, B alive & A alive, B dead & A dead, B alive & A, B dead \\
\hline A, B alive & $1-\left(g_{A}+g_{B}\right)$ & $g_{B}$ & $g_{A}$ & 0 \\
A alive, B dead & 0 & $1-f_{B}$ & 0 & $f_{B}$ \\
A dead, B alive & 0 & 0 & $1-f_{A}$ & $f_{A}$ \\
A, B dead & 0 & 0 & 0 & 1 \\
\hline
\end{tabular}

In each case, we have to compute the prices of the products (the two CDS and the CD). We summarize the different results in the following table:

\begin{tabular}{|c|ccc|}
\hline & $C D S_{t}^{A}$ & $C D S_{t}^{B}$ & $C D_{t}$ \\
\hline A, B alive & $g_{A}+g_{B} f_{B}$ & $g_{B}+g_{A} f_{A}$ & $g_{A}\left(F_{1}+f_{A} F_{2}\right)+g_{B}\left(F_{1}+f_{B} F_{2}\right)$ \\
A alive, B dead & $f_{B}$ & 0 & $f_{B} F_{2}$ \\
A dead, B alive & 0 & $f_{A}$ & $f_{A} F_{2}$ \\
A, B dead & 0 & 0 & 0 \\
\hline
\end{tabular}

For example, when $B$ is already in default (that is $\tau_{B}<t \leq T \wedge \tau_{A}$ ), the $C D S^{B}$ has generated a cash-flow of 1 at $\tau_{B}$ and therefore its price is zero. Likewise, the credit derivative $C D$ has generated a cash-flow of $F_{1}$ at $\tau_{B}$ and therefore its price depends on $F_{2}$ only.

Now we try to find the replicating portfolio to hedge the $C D$. This portfolio contains the two CDS and some cash. In the next table, we introduce the hedging ratios:

\begin{tabular}{|c|ccc|}
\hline & $C D S_{t}^{A}$ & $C D S_{t}^{B}$ & cash \\
\hline A, B alive & $\delta^{A}$ & $\delta^{B}$ & $\operatorname{cash}^{B}$ \\
A alive, B dead & $\delta^{B, A}$ & 0 & $\operatorname{cash}^{B}$ \\
A dead, B alive & 0 & $\delta^{A, B}$ & $\operatorname{cash}^{A}$ \\
A, B dead & 0 & 0 & $\operatorname{cash}^{A, B}$ \\
\hline
\end{tabular}

\footnotetext{
${ }^{3}$ In the following computations, we remove the explicit reference to the time. All these joint probabilities can be made explicit by using the copula.

${ }^{4}$ In the copula model, there cannot be simultaneous defaults since $\mathbb{Q}\left(\tau_{A}=\tau_{B}\right)=0$.

${ }^{5}$ This matrix cannot mislead the reader, since the default contagion process is not Markov.
} 
By definition, the initial mark-to-market of the self-financing portfolio is 0 , and it will remain unchanged over time in all cases. Using the two preceding tables, it is straightforward to obtain the following linear system for the ratios:

$$
\begin{aligned}
& \delta^{A}\left(g_{A}+g_{B} f_{B}\right)+\delta^{B}\left(g_{B}+g_{A} f_{A}\right)+g_{A}\left(F_{1}+f_{A} F_{2}\right)+g_{B}\left(F_{1}+f_{B} F_{2}\right) \quad+\text { cash }=0 \\
& \delta^{A, B} f_{A}+f_{A} F_{2}+\operatorname{cash}^{A}=0 \\
& \delta^{B, A} f_{B}+f_{B} F_{2}+\operatorname{cash}^{B}=0 \\
& \operatorname{cash}^{A, B}=0 \text {. }
\end{aligned}
$$

Here we have only four equations to find eight unknown parameters. The four remaining equations are given by the self-financing condition. We can interpret them as continuity conditions for the value of portfolio at the successive default times.

$$
\begin{cases}\operatorname{cash}^{A}=\operatorname{cash}+F_{1}+\delta^{A}+\left(\delta^{B}-\delta^{A, B}\right) f_{A} & \text { first-to-default is A, } \\ \operatorname{cash}^{B}=\operatorname{cash}+F_{1}+\delta^{B}+\left(\delta^{A}-\delta^{B, A}\right) f_{B} & \text { first-to-default is B, } \\ \operatorname{cash}^{A, B}=\operatorname{cash}^{A}+F_{2}+\delta^{A, B} & \text { second-to-default is B, } \\ \operatorname{cash}^{A, B}=\operatorname{cash}^{B}+F_{2}+\delta^{B, A} & \text { second-to-default is A. }\end{cases}
$$

Solving this system, we find:

$$
\begin{aligned}
\delta^{A} & =-\frac{\left(1-f_{A}\right) F_{1}+f_{A}\left(1-f_{B}\right) F_{2}}{1-f_{A} f_{B}} \\
\delta^{B} & =-\frac{\left(1-f_{B}\right) F_{1}+f_{B}\left(1-f_{A}\right) F_{2}}{1-f_{A} f_{B}} \\
\delta^{A, B} & =-F_{2} \\
\delta^{B, A} & =-F_{2},
\end{aligned}
$$

and the cash is always zero

$$
\operatorname{cash}=\operatorname{cash}^{A}=\operatorname{cash}^{B}=\operatorname{cash}^{A, B}=0 .
$$

Now, as in the last section, we are going to compute the derivative of the value of the replicating portfolio with respect to the correlation parameter $\rho$.

For a (smooth) function $h$, we denote:

$$
h^{\prime}=\left.\frac{\partial h}{\partial \rho}\right|_{C D S^{A} \text { and } C D S^{B} \text { unchanged }} .
$$

We then have for $t<\tau_{A}, t<T, t<\tau_{B}$ (no default):

$$
\begin{aligned}
0 & =g_{A}^{\prime}+g_{B}^{\prime} f_{B}+g_{B} f_{B}^{\prime} \\
0 & =g_{B}^{\prime}+g_{A}^{\prime} f_{A}+g_{A} f_{A}^{\prime} \\
& =\left(g_{A}^{\prime}+g_{B}^{\prime}\right)\left(F_{1}-F_{2}\right) \\
& =-\frac{\left(1-f_{B}\right) g_{A} f_{A}^{\prime}+\left(1-f_{A}\right) g_{B} f_{B}^{\prime}}{1-f_{A} f_{B}}\left(F_{1}-F_{2}\right)
\end{aligned}
$$




$$
\frac{\partial \text { portfolio }}{\partial \rho} \mid \begin{gathered}
C D S^{A} \text { and } C D S^{B} \text { unchanged } \\
\text { portfolio composition unchanged }
\end{gathered}=-\frac{\left(1-f_{B}\right) g_{A} f_{A}^{\prime}+\left(1-f_{A}\right) g_{B} f_{B}^{\prime}}{1-f_{A} f_{B}}\left(F_{1}-F_{2}\right) .
$$

As in the previous section, we suppose that the trader prices and hedges his portfolio using a parameter $\hat{\rho}$, while the "true" parameter is $\rho=\hat{\rho}+\Delta \rho$.

We study the case $A$ defaults before $B$, and before time $T$.

At $\tau_{A}$, the portfolio is composed of:

$$
\begin{aligned}
& -C D \\
& \text { - } \delta^{B}(\hat{\rho}) C D S^{B} \\
& \text { - } \underbrace{\text { cash }}_{\text {initial cash }}+\underbrace{F_{1}+\delta^{A}(\hat{\rho})}_{\text {default cash-flows }} \text { of cash. }
\end{aligned}
$$

After rebalancing the hedge, it will be composed of:

$$
\begin{aligned}
& \text { - the } C D \\
& \bullet \delta^{A, B}(\rho) C D S^{B} \\
& \text { - } \underbrace{\text { cash }}_{\text {initial cash }}+\underbrace{F_{1}+\delta^{A}(\hat{\rho})}_{\text {default cash-flows }}+\underbrace{\left(\delta^{B}(\hat{\rho})-\delta^{A, B}(\rho)\right) f_{A}(\rho)}_{\text {rebalancing cost }} \text { of cash. }
\end{aligned}
$$

The Profit \& Loss at time $T$ after a default of $A$ will not depend on the occurrence of a default of $B$, it is then:

$$
\begin{aligned}
\widehat{P \& L}_{T}^{A} & =F_{2} f_{A}(\rho)+\delta^{A, B}(\rho) f_{A}(\rho)+\operatorname{cash}+F_{1}+\delta^{A}(\hat{\rho})+\delta^{B}(\hat{\rho}) f_{A}(\rho)-\delta^{A, B}(\rho) f_{A}(\rho) \\
& =F_{2} f_{A}(\rho)+\operatorname{cash}+F_{1}+\delta^{A}(\hat{\rho})+\delta^{B}(\hat{\rho}) f_{A}(\rho) \\
& =\left(F_{2+} \delta^{B}(\hat{\rho})\right)\left(f_{A}(\rho)-f_{A}(\hat{\rho})\right) \\
& =\left(F_{2+} \delta^{B}(\hat{\rho})\right) f_{A}^{\prime} \Delta \rho+o(\Delta \rho) \\
& =-\frac{\left(1-f_{B}\right)}{1-f_{A} f_{B}} f_{A}^{\prime}\left(F_{1}-F_{2}\right) \Delta \rho+o(\Delta \rho)
\end{aligned}
$$

We also have by symmetry between A and B:

$$
\widehat{P \& L}_{T}^{B}=-\frac{\left(1-f_{A}\right)}{1-f_{A} f_{B}} f_{B}^{\prime}\left(F_{1}-F_{2}\right) \Delta \rho+o(\Delta \rho) .
$$

Knowing the sign of $f_{A}^{\prime}, f_{B}^{\prime}$ and $F_{1}-F_{2}$, we have the sign of the errors.

We observe that the expectation of $\widehat{P \& L}_{T}^{A}$ under $\mathbb{P}$ has now a very simple expression:

$$
\begin{aligned}
\mathbb{E}^{\mathbb{Q}}\left[P \& L_{T}\right] & =\mathbb{P}\left[\tau_{A}<T, \tau_{A}<\tau_{B}\right] \widehat{P \& L}_{T}^{A}+\mathbb{P}\left[\tau_{B}<T, \tau_{B}<\tau_{A}\right] \widehat{P \& L}_{T}^{B} \\
& =-g_{A} \frac{\left(1-f_{B}\right)}{1-f_{A} f_{B}} f_{A}^{\prime}\left(F_{1}-F_{2}\right) \Delta \rho-g_{B} \frac{\left(1-f_{A}\right)}{1-f_{A} f_{B}} f_{B}^{\prime}\left(F_{1}-F_{2}\right) \Delta \rho+o(\Delta \rho) \\
& =\frac{\partial p o r t f o l i o}{\partial \rho} \mid \begin{array}{c}
C D S^{A} \text { and } C D S^{B} \text { unchanged } \\
\text { portfolio composition unchanged }
\end{array}
\end{aligned}
$$


We can conclude and state:

Approximation result 3.1. We denote $H_{A}$ and $H_{B}$ :

$$
\left\{\begin{array}{l}
H_{A}:=-\frac{\left(1-f_{B}\right)}{1-f_{A} f_{B}} f_{A}^{\prime}\left(F_{1}-F_{2}\right) \\
H_{B}:=-\frac{\left(1-f_{A}\right)}{1-f_{A} f_{B}} f_{B}^{\prime}\left(F_{1}-F_{2}\right)
\end{array}\right.
$$

and $g_{A}^{\mathbb{P}}$ and $g_{B}^{\mathbb{P}}$ :

$$
\left\{\begin{array}{c}
g_{A}^{\mathbb{P}}(\hat{\rho}):=\mathbb{P}\left[\tau_{A}<T, \tau_{A}<\tau_{B}\right] \\
g_{B}^{\mathbb{P}}(\hat{\rho}):=\mathbb{P}\left[\tau_{B}<T, \tau_{B}<\tau_{A}\right]
\end{array}\right.
$$

Then:

$$
\mathbb{E}^{\mathbb{P}}\left[P \& L_{T}\right] \simeq\left(g_{A}^{\mathbb{P}}(\hat{\rho}) H_{A}+g_{B}^{\mathbb{P}}(\hat{\rho}) H_{B}\right)\left(\mathbb{E}^{\mathbb{P}}[\rho]-\hat{\rho}\right) .
$$

And if in addition we suppose $\rho$ Gaussian under the historical probability, centered around $\hat{\rho}$, the value used by the trader, and of standard deviation $\sigma(\rho)$ :

$$
\begin{aligned}
\sigma^{\mathbb{P}}\left[P \& L_{T}\right] & =\sqrt{\mathbb{E}^{\mathbb{P}}\left[P \& L_{T}^{2}\right]} \\
& \simeq \sqrt{\left(g_{A}^{\mathbb{P}}(\hat{\rho}) H_{A}^{2}+g_{B}^{\mathbb{P}}(\hat{\rho}) H_{B}^{2}\right)} \sigma^{\mathbb{P}}(\rho) .
\end{aligned}
$$

If we now suppose that the two credits have the same spreads (more precisely $f_{A}=f_{B}=f, g_{A}=g_{B}=g$, $\left.f_{A}^{\mathbb{P}}=f_{B}^{\mathbb{P}}=f^{\mathbb{P}}, g_{A}^{\mathbb{P}}=g_{B}^{\mathbb{P}}=g^{\mathbb{P}}\right)$ :

$$
\begin{aligned}
\sigma^{\mathbb{P}}\left[P \& L_{T}\right] & \simeq \frac{1}{\sqrt{2}} \frac{\sqrt{g^{\mathbb{P}}}}{g}\left|\frac{\partial \text { portfolio }}{\partial \rho}\right| \begin{array}{c}
C D S^{A} \text { and } C D S^{B} \text { unchanged } \\
\text { portfolio composition unchanged }
\end{array} \mid \sigma^{\mathbb{P}}(\rho) \\
& \simeq \frac{1}{\sqrt{2}} \frac{\sqrt{\mathbb{P}\left[\tau_{A}<T, \tau_{A}<\tau_{B}\right]}}{\mathbb{Q}\left[\tau_{A}<T, \tau_{A}<\tau_{B}\right]} \\
& \left|\frac{\partial p o r t f o l i o}{\partial \rho}\right| \begin{array}{c}
C D S^{A} \text { and } C D S^{B} \text { unchanged } \\
\text { portfolio composition unchanged }
\end{array} \mid \sigma^{\mathbb{P}}(\rho)
\end{aligned}
$$

We now turn to a few examples. First, when the default times are independent with a common and constant intensity $\lambda:=\lambda^{A}=\lambda^{B}$, we can write:

$$
\begin{aligned}
\mathbb{P}\left[\tau_{A}<T, \tau_{A}<\tau_{B}\right] & =\int_{0}^{+\infty} \mathbb{P}\left[\tau_{A}<T, \tau_{A}<s \mid \tau_{B}=s\right] \lambda \mathrm{e}^{-\lambda s} \mathrm{~d} s \\
& =\int_{0}^{+\infty}\left(1-\mathrm{e}^{-\lambda(T \wedge s)}\right) \lambda \mathrm{e}^{-\lambda s} \mathrm{~d} s \\
& =\frac{1}{2}\left(1-\mathrm{e}^{-2 \lambda T}\right)
\end{aligned}
$$

and if $\lambda T \ll 1$,

$$
\mathbb{P}\left[\tau_{A}<T, \tau_{A}<\tau_{B}\right] \simeq \lambda T \simeq \mathbb{P}\left[\tau_{A}<T\right]
$$


We deduce then:

$$
\sigma^{\mathbb{P}}\left[P \& L_{T}\right] \simeq \frac{1}{\sqrt{2}} \frac{\sqrt{\mathbb{P}[\tau \leq T]}}{\mathbb{Q}[\tau \leq T]}\left|\frac{\partial p o r t \text { folio }}{\partial \rho}\right| \begin{gathered}
C D S^{A} \text { and } C D S^{B} \text { unchanged } \\
\text { portfolio composition unchanged }
\end{gathered} \mid \sigma^{\mathbb{P}}(\rho) .
$$

Now when the defaults are not independent, using the copula we have:

$$
\begin{aligned}
\mathbb{P}\left[\tau_{A}<T, \tau_{A}<\tau_{B}\right] & =\int_{0}^{+\infty} \mathbb{P}\left[\tau_{A}<T \wedge s \mid \tau_{B}=s\right] \mathbb{P}\left[\tau_{B} \in \mathrm{d} s\right] \\
& =1-\int_{0}^{+\infty} \partial_{2} C\left(\mathbb{P}\left[\tau_{A}>T \wedge s\right], \mathbb{P}\left[\tau_{B}>s\right]\right) \mathbb{P}\left[\tau_{B} \in \mathrm{d} s\right]
\end{aligned}
$$

If the probability of a default is low, as $C(x, y) \sim C_{\perp}(x, y)$, if the default probability is relatively low,

$$
\begin{aligned}
& x \rightarrow 1 \\
& y \rightarrow 1
\end{aligned}
$$

we find back the same result than in the independent case.

\subsection{Case of $d$ credits: 1st discussion (degenerated correlation matrix)}

We now study the case of $d$ credits. We suppose that the first default will reveal the "true" correlation parameter. This means that at the first default, we will have a jump of $P \& L$ because it will not have been correctly immunized against the market movement which occurs, and because after the 'true' correlation parameter is revealed, we have a jump of the MtM of the $C D$.

We suppose that all credits are identical, and we take the same notations than in the previous section. We therefore have:

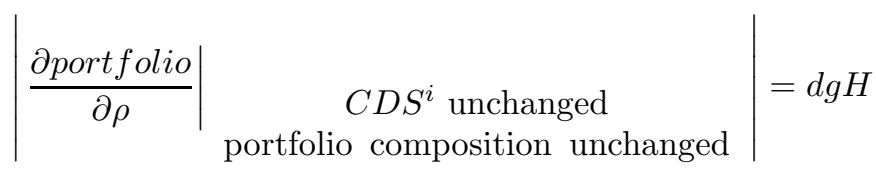

and:

$$
\mathbb{E}^{\mathbb{P}}\left[P \& L_{T}\right] \simeq d g^{\mathbb{P}} H\left(\mathbb{E}^{\mathbb{P}}[\rho]-\hat{\rho}\right) .
$$

If we suppose that the correlation coefficient used to hedge the portfolio is equal to $\mathbb{E}^{\mathbb{P}}[\rho]$,

$$
\begin{aligned}
\mathbb{E}^{\mathbb{P}}\left[P \& L_{T}\right] & \simeq 0 \\
\sigma^{\mathbb{P}}\left[P \& L_{T}\right] & \simeq \sqrt{\left(d g^{\mathbb{P}} H^{2}\right)} \sigma(\rho) \\
& \simeq \frac{1}{\sqrt{d} \frac{\sqrt{\mathbb{P}\left[\tau_{1}<T, \tau_{1}<\tau_{2} \wedge \ldots \wedge \tau_{d}\right]}}{\mathbb{Q}\left[\tau_{1}<T, \tau_{1}<\tau_{2} \wedge \ldots \wedge \tau_{d}\right]}} \\
& \left|\frac{\partial \text { portfolio }}{\partial \rho}\right| \begin{array}{c}
C D S^{i} \text { unchanged } \\
\text { portfolio composition unchanged }
\end{array} \mid \sigma^{\mathbb{P}}(\rho) .
\end{aligned}
$$




\subsection{Case of $d$ credits: 2nd discussion (non-degenerated correlation matrix)}

We now study the case of $d$ credits. The correlations are defined pairwise. The error on each correlation coefficient can be written as in the 2 underlying credits case. If we assume that the different partial derivatives $\frac{\partial p o r t f o l i o}{\partial \rho_{i, j}} \mid \quad C D S^{k}$ unchanged have the same sign, that the errors on the each correlation paportfolio composition unchanged

rameter have the same standard deviation, denoted $\sigma(\rho)$, and even have a correlation of 1 (this means that the errors are the same), we approximate:

$$
\mathbb{E}^{\mathbb{P}}\left[P \& L_{T}\right] \simeq 0
$$

and:

$$
\sigma^{\mathbb{P}}\left[P \& L_{T}\right] \simeq \frac{1}{\sqrt{2}} \frac{\sqrt{\mathbb{P}[\tau \leq T]}}{\mathbb{Q}[\tau \leq T]}\left|\frac{\partial p o r t \text { folio }}{\partial \rho}\right| \begin{gathered}
C D S^{k} \text { unchanged } \\
\text { portfolio composition unchanged }
\end{gathered} \mid \sigma^{\mathbb{P}}(\rho)
$$

where $\frac{\partial \text { portfolio }}{\partial \rho} \mid$ is computed by shifting simultaneously all the correlation coefficients.

\section{Conclusion}

We have studied here the consequence, for a trader, of a misspecification of some parameters introduced in an model for exotic credit derivatives (recovery rates, correlation coefficients). The results we obtained are quite similar, but of course with less importance, to those obtained by El Karoui, Jeanblanc-Picqué and Shreve [4].

We have also discussed the consequence of hedging a credit derivative instrument against small CDS margin movements, while the risk on which most of the model is based are default risk. This point is not only theoretical: we find some traders or structuring teams, aware of that point, who will introduce a cancellation option in their complex products in order to avoid a negative theta effect in the hedging.

\section{REFERENCES}

[1] L. Andersen and J. Sidenius, Extensions to the Gaussian copula: random recovery and random factor loadings. J. Credit Risk 1 (2004) 29-70.

[2] T. Bielecki and M. Jeanblanc, Pricing and Hedging of credit risk: replication and mean-variance approaches. Working paper (2003).

[3] B. Dupire, Pricing with a smile. Risk 7 (1994) 18-20.

[4] N. El Karoui, M. Jeanblanc-Picqué and S.E. Shreve, Robustness of the Black and Scholes formula. Math. Fin. 8 (1998) $93-126$.

[5] M. Jeanblanc and M. Rutkowski, Hedging of credit derivatives within the reduced-form framework. Working paper (2003).

[6] D. Lando, On Cox processes and credit-risky securities. Rev. Derivatives Res. 2 (1998) 99-120.

[7] P. Schönbucher and D. Schubert, Copula-dependent default risk in intensity models. ETH Zurich, working paper (2001). 